\title{
Control of the Wireless Microrobot with Multi-DOFs Locomotion for Medical Applications
}

\author{
Takuya Okada, Shuxiang Guo, Nan Xiao, Fu Qiang and Yasuhiro Yamauchi \\ Dept. of Intelligent Mechanical Systems Eng'g \\ Kagawa University, Japan \\ 2217-20, Hayashi-cho, Takamatsu, 761-0396, Japan \\ tttokada1217@gmail.com, \{guo \& xiao\}@eng.kagawa-u.ac.jp, \{s12g528 \& s12g535\}@stmail.eng.kagawa-u.ac.jp
}

\begin{abstract}
In this paper, we discuss a multi-DOFs locomotion and position detection system of the microrobot to realize the wireless locomotion. Also we propose one wireless microrobot with two motions controlled by changing the outside magnetic field. When generating alternating magnetic field, the microrobot can be driven by the fin motion just like fish. When generating rotating magnetic field, the microrobot can move in the spiral jet motion. Based on the experimental results of the locomotion, we confirm that the microrobot can move in forward, backward, floating motion and turning motion. In addition, we have developed a position detection system of the microrobot. We use least square method to solve the inverse problem. To evaluate the error of the proposed position detection system, we will do experiment by using 6 magnetic field sensors and program. Base on this experiment, we also confirm that the proposed position detection system can detect the position of the microrobot within $50 \mathrm{~mm}$ from sensor in vertical direction, and its average error is within $6.09 \mathrm{~mm}$.
\end{abstract}

Index Terms - position detection system, wireless robot, magnetic robot, multi-DOFs locomotion

\section{INTRODUCTION}

Microrobot has been great developed in the world today. Various studies have been made for the purpose to drive the robot in a pipe. For example, medical robots are used in driving in the human body and the robots are used in the plant maintenance and so on have been developed [1]-[7]. In recent researches, some of these robots can wireless controlled by outside magnetic field [8]-[14]. Three-Dimensional Electromagnetic Actuation System for Intravascular Locomotion has been developed [15]-[19]. It is necessary to know where these robots are located in a magnetic field. However, to detect specific position of the microrobot is very difficult because of invisibility in the pipe. In this study, we have embarked on a method for detecting the position of the robot from an external non-contact manner.

Recent years, X-ray is used the as a way of knowing the body, such as inside information. This method has the drawback that scale of the device is large and adversely affects to the surrounding environment. Therefore, the development of small position detection system has been obtained in safety. Then, position detection system has been developed, and now research is indicated in following. The position detection method, there is a way to detect the magnetic field of the magnet inside the robot using a Hall element [20], [21]. The Hall element has the disadvantage to large changes in temperature and it is unsuitable for the sensitivity dependent on the temperature. Moreover, it has also been reported threedimensional position detection system using a flux-gate magnetic sensor [22]. This detection system can also detect very small magnetic field, but it affects earth magnetic field. Average error of this sensor device is $2.10 \mathrm{~mm}$. In addition to the study of position detection system using Extreme low frequency (ELF) electromagnetic emitter and magnetic field sensor has been developed [23]. This system is very expensive for using the ELF electromagnetic emitter to generate a magnetic field. And, it can be transmitted into the steel pipe. Average motion of the tracking error of this sensor device is $18.5 \mathrm{~cm}$.

This paper discussed multi-DOFs locomotion and position detection system of the microrobot to realize the wireless locomotion. Proposed wireless microrobot has two motions by changing the outside magnetic field. When it generates alternating magnetic field, the microrobot can move in the fin motion just like fish. When generating rotating magnetic field, the microrobot can move by the spiral jet motion. According to experimental results of the locomotion, we confirmed that the microrobot can move forward and backward. It can also move by floating motion and turning motion. In addition, we developed position detection system of the microrobot. This position detection system uses intensity of the magnetic field which is generated from the $\mathrm{O}$ ring magnet. We used least square method to solve the inverse problem. When equation function of the least square method is the smallest of all, we chose 6 parameters of that time which are $\mathrm{x}, \mathrm{y}, \mathrm{z}$, roll, pitch and yaw as output of the position parameter.

This paper is structured as the following. Firstly, we propose the configuration of the control systems and mechanism of the microrobot. Secondly, we discussed specification and characteristic of the microrobot. Thirdly, we will do the experiment of the multi-DOFs locomotion of the microrobot. To realize the multi-DOFs locomotion, we confirmed the state of the microrobot which is turning right and turning left by experiment in the pipe filled with water. Fourthly, we explained the principle and mechanism of magnetic position detection system using high sensitive magnetic field sensor. And then, we show the results of effectiveness and experimental verification. 


\section{CONFIGURATION OF THE CONTROL SYSTEMS}

\section{A. Proposed Robot Control Systems}

To locomotion and control of the microrobot, we developed robot control systems. This system is shown in Fig. 1. Locomotion system of the microrobot is the right part in Fig. 1. Position detection system of the microrobot is the left part in Fig. 1. Locomotion system is consisted of large 3 axes Helmholtz coil system and the microrobot. The microrobot can move by outside magnetic field which is generated by 3 axes Helmholtz coil system. Moving direction of the microrobot can change by direction of the outside magnetic field. Moving speed of the microrobot can change by frequency of the outside magnetic field. It is possible to generate 3D magnetic field by 3 axes Helmholtz coil system. Because of this, Multi-DOFs locomotion of the microrobot was realized. In additionally, we designed and developed position detection system of the microrobot. It is possible to know the positioning information of the microrobot where the microrobot locates in the coil system now. We used 6 magnetic sensors to measure the magnetic field were generated by magnet in the body. By using locomotion system and detection system, we hope that wireless microrobot which is moving in the liquid will be realized.

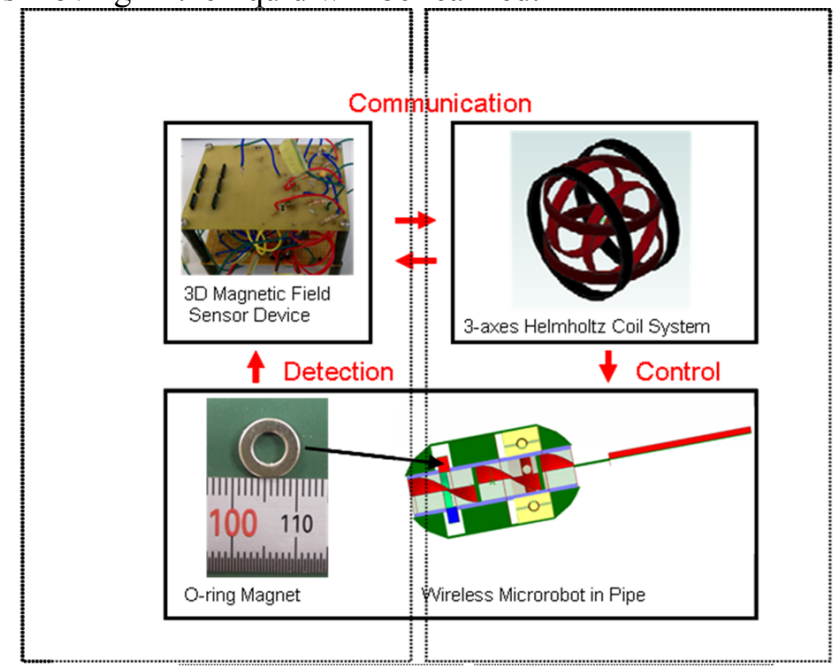

Position detection system

Locomotion system

Fig. 1 Configuration of the system

\section{B. Specification of the Hybrid Microrobot}

Developed hybrid microrobot is shown in Fig. 2 and its specification is shown in Table I. This microrobot has two motion mechanisms. It is possible to move just like fish by vibrating its body. And, it is possible to move by rotating its body. These two motions can realize by switching direction of the outer magnetic field. With the outside alternating magnetic field, the microrobot moves by fish like motion. And, with the outside rotating magnetic field, the microrobot moves by spiral jet motion. The main propulsion is the fin motion due to stable motion and high propulsive force. The spiral jet motion takes advantage of motion in the complex area.

\section{Fin Motion Spiral Jet Motion}

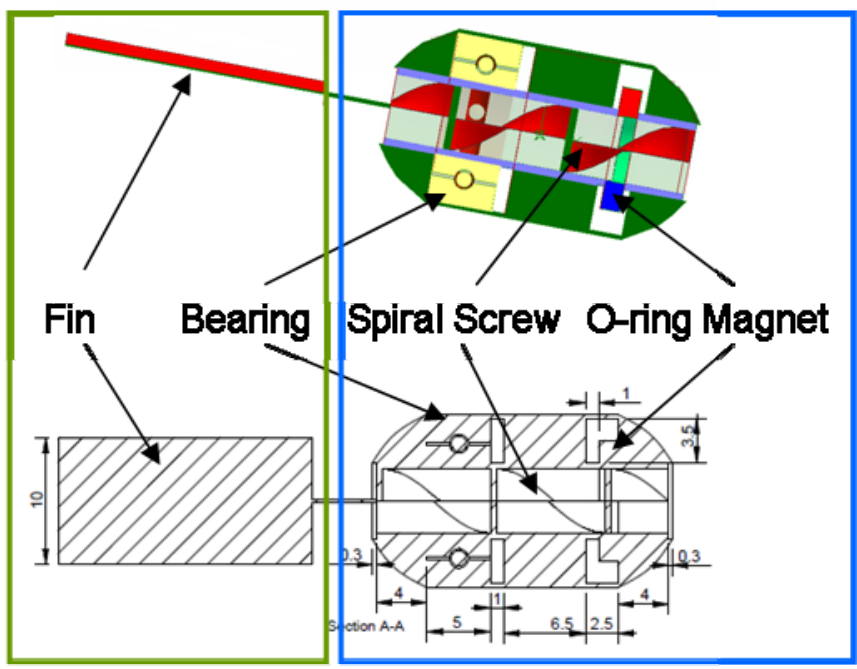

Fig. 2 Proposed hybrid type microrobot

TABLE I

Specification of the hybrid microrobot

Size of the robot $(\mathrm{mm})$

Size of the fin $(\mathrm{mm})$

Total length (include fin) ( $\mathrm{mm}$ )

Weight of the robot (g)

Size of the O-ring magnet $(\mathrm{mm})$

Residual magnetic flux density $(\mathrm{mT})$

Size of the screw $(\mathrm{mm})$

Pitch of the screw (mm) $\phi 15 \times 28$

$10 \times 20 \times 0.1$

56

3.56

$\phi 9.5 \times \phi 5.1 \times 1.5$

1250

$\phi 5 \times 23 \times 0.5$

18

\section{EXPERIMENTAL RESULTS OF THE 3 DOFS LOCOMOTION}

We evaluated moving speed of the microrobot. This result is shown in Fig. 3. This result indicated that the microrobot has motion of the wide range frequency. When the frequency is low, the fin motion is superior to the spiral jet motion. And, maximum speed of the fin motion is $7 \mathrm{~mm} / \mathrm{s}$ at $14 \mathrm{~Hz}$. When the frequency is high, the spiral jet motion is superior to the fin motion.

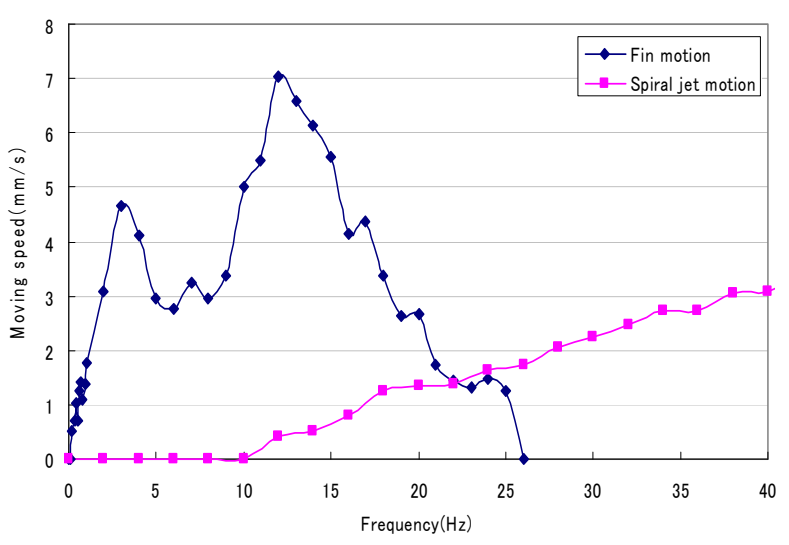

Fig. 3 Characteristic evaluations of the moving speed 
State of the microrobot which is moving is shown in Fig. 4 - Fig. 6. Fig. 4 is state of the microrobot which is moving forward.

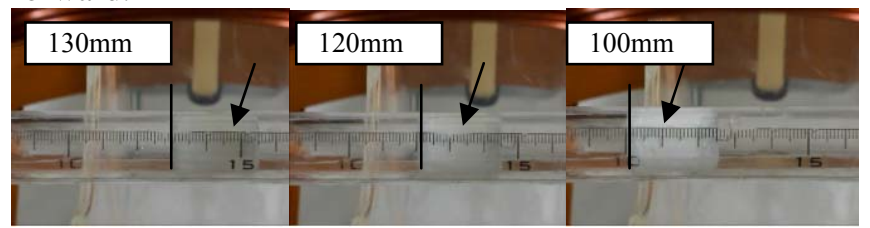

(a) at $0 \mathrm{~s}$

(b) at $3 \mathrm{~s}$

(c) at $10 \mathrm{~s}$

Fig. 4 State of the microrobot which is moving forward

Fig. 5 is state of the microrobot which is moving backward. This motion can realize by the spiral jet motion.

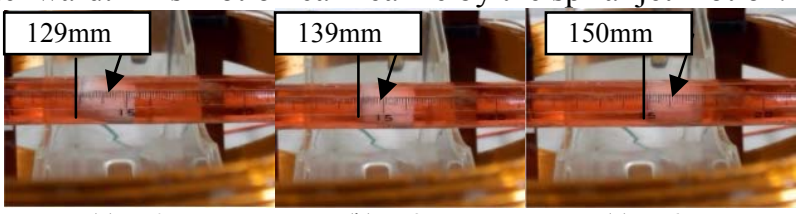
(a) at $0 \mathrm{~s}$
(b) at $3 \mathrm{~s}$
(c) at $6 \mathrm{~s}$
Fig. 5 State of the microrobot which is moving backward

Fig. 6 is state of the microrobot which is moving in vertical direction. This microrobot has neutral buoyancy. Because of this, the microrobot also moved in vertical direction by equal speed.

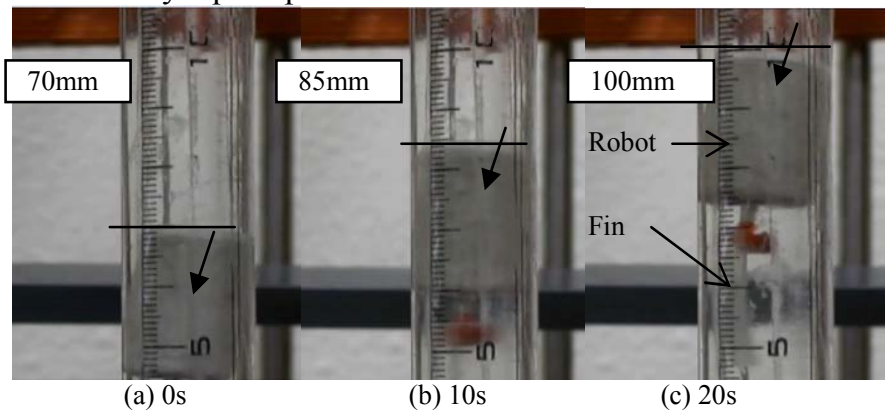

Fig. 6 State of the microrobot which is moving in vertical direction

We did experiment of changing course of the microrobot to evaluate multi-DOFs locomotion. Experimental states of the microrobot which is turning left are shown in Fig. 7.

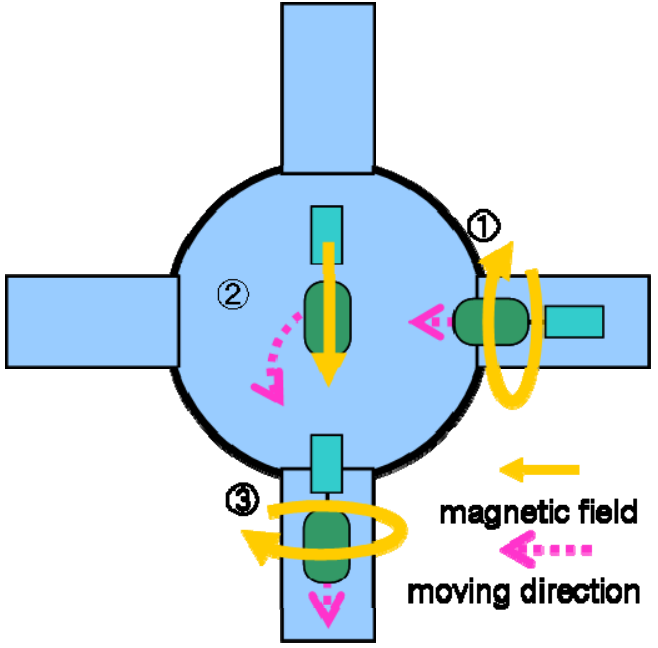

Fig. 7 Outline of the experiment (turn left)
Experimental results of the microrobot which is turning left are shown in Fig. 8. This experiment was done in the water. Firstly, the microrobot moves toward the center from right side of the picture. Secondly, the microrobot turns left to proceed to the front side of the picture. Finally, the microrobot moves toward the front side of the picture by rotating motion or fin motion. Thus, we confirmed that the microrobot can turn left and proceed toward another pipe line.

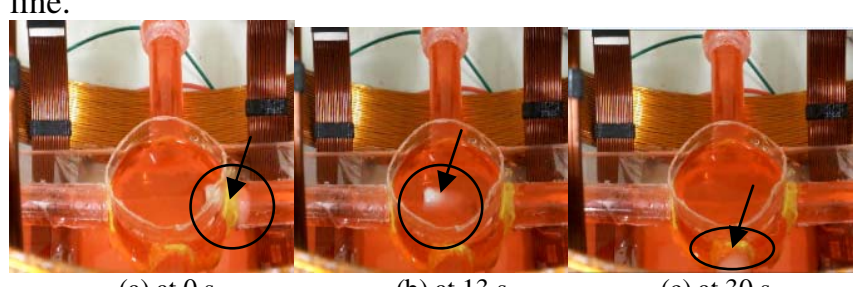

(a) at $0 \mathrm{~s}$

(b) at $13 \mathrm{~s}$

(c) at $30 \mathrm{~s}$

Fig. 8 Experimental results of the microrobot which is turning left

Experimental states of the microrobot which is turning right are shown in Fig. 9.

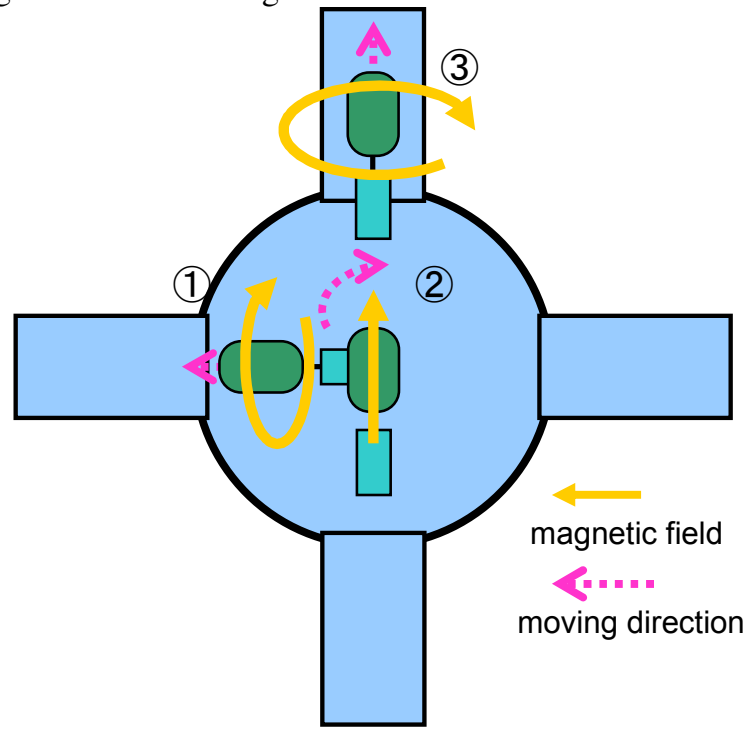

Fig. 9 Outline of the experiment (turn right)

Experimental results of the microrobot which is turning right are shown in Fig. 10. Firstly, the microrobot moves toward the center from right side of the picture. Secondly, the microrobot turns left to proceed to the back side of the picture. Finally, the microrobot moves toward the back side of the picture by rotating motion or fin motion. Thus, we confirmed that the microrobot can turn right and proceed toward the back side pipe line. Based on total experimental results, we realized the 3 DOFs locomotion of the microrobot.

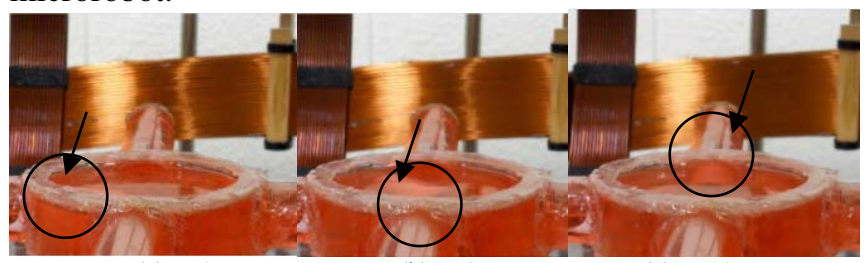

(a) at $0 \mathrm{~s}$

(b) at $2 \mathrm{~s}$

(c) at $10 \mathrm{~s}$

Fig. 10 Experimental results of the microrobot which is turning right 


\section{THE PRINCIPLE OF POSITION DETECTION METHOD}

\section{A. Position detection system}

To measure the position and posture of the microrobot, we used solving inverse problem. Firstly, we analyzed magnetic field which is generated by magnet in the body of the microrobot. Secondly, we measured that magnetic field by using six magnetic sensors. Thirdly, we solved the inverse problems and used least square method between theoretical results and experimental results of the magnetic flux density. Proposed position detection system is shown in Fig. 11. The array of six magnetic field sensors are $2 \times 3$ matrices to implement stable measurement.

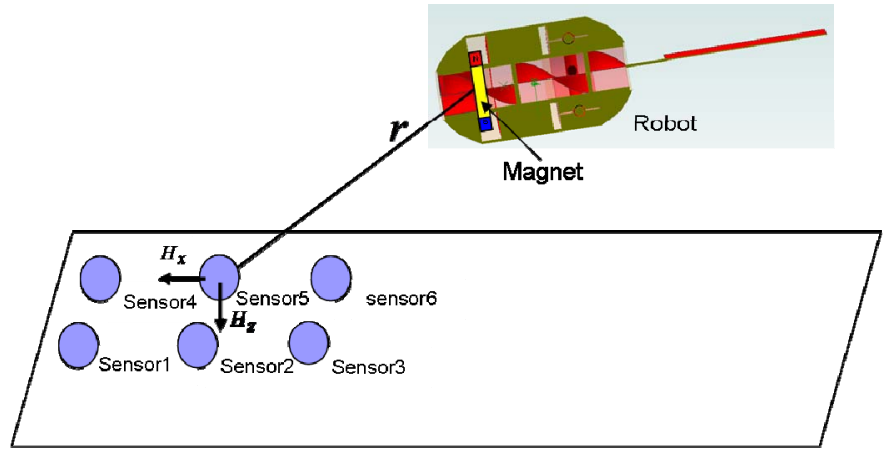

Fig. 11 Position detection system of the microrobot

\section{B. Analysis of the magnetic field}

We analyzed magnetic flux density of the magnet in the body of the microrobot. We made model of the intensity of magnetic field based on electromagnetism. This equation is indicated in $\mathrm{Eq}(1)-(6) . \mathrm{Eq}(4), \mathrm{Eq}(5)$ and $\mathrm{Eq}(6)$ is used to analyze the magnetic field. Model of the magnetic field analysis method is shown in Fig. 12.

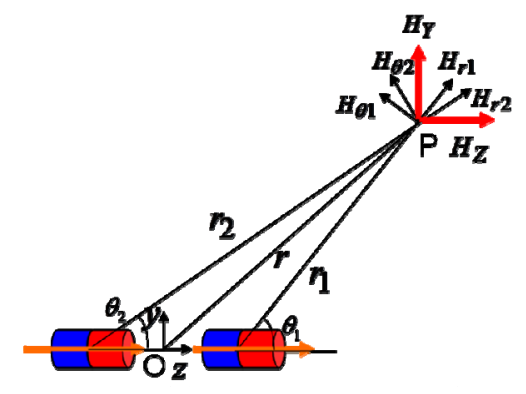

magnetic dipole moment 2

magnetic dipole moment 1

Fig. 12 Model of the magnetic field analysis method

$$
\begin{gathered}
U=\frac{m l \cos \theta}{4 \pi \mu_{0} r^{2}} \\
H_{r}=-\frac{\partial U}{\partial r}=\frac{m l}{2 \pi \mu_{0} r^{3}} \cos \theta \\
H_{\theta}=-\frac{\partial U}{r \partial \theta}=\frac{m l}{4 \pi \mu_{0} r^{3}} \sin \theta
\end{gathered}
$$

$$
H_{X}=\frac{X}{\sqrt{X^{2}+Y^{2}}}\left(\frac{3 m l}{4 \pi \mu_{0} r_{1}^{3}} \sin \theta_{1} \cos \theta_{1}+\frac{3 m l}{4 \pi \mu_{0} r_{2}^{3}} \sin \theta_{2} \cos \theta_{2}\right)
$$

$$
\begin{aligned}
& H_{Y}=\frac{Y}{\sqrt{X^{2}+Y^{2}}}\left(\frac{3 m l}{4 \pi \mu_{0} r_{1}^{3}} \sin \theta_{1} \cos \theta_{1}+\frac{3 m l}{4 \pi \mu_{0} r_{2}^{3}} \sin \theta_{2} \cos \theta_{2}\right) \\
& H_{Z}=\frac{m l}{4 \pi \mu_{0} r_{1}^{3}}\left(2 \cos ^{2} \theta_{1}-\sin ^{2} \theta_{1}\right)+\frac{m l}{4 \pi \mu_{0} r_{2}^{3}}\left(2 \cos ^{2} \theta_{2}-\sin ^{2} \theta_{2}\right)
\end{aligned}
$$

Where $U$ is the magnetic potential, $\mathrm{m}$ is the magnetic moment, $l$ is the distance between the magnetic poles, $r$ is the distance between measurement position and magnet, $\theta$ is the angle between measurement position and magnet. $\mu_{0}$ is the permeability of vacuum. $L$ is the half length between two magnetic potentials of the magnet. $H_{r}$ is intensity of magnetic field in $\mathrm{r}$ direction, and $H_{\theta}$ is intensity of magnetic field in $\theta$ direction.

Analytical results of the magnetic field based on these equations are shown in Fig. 13 - 15. Fig. 13 is one magnet, Fig. 14 is four magnets, Fig. 15 is o-ring type magnet, respectively. This result indicated that the magnet having multi magnetic potentials is higher magnetic field area than one magnetic potential type.

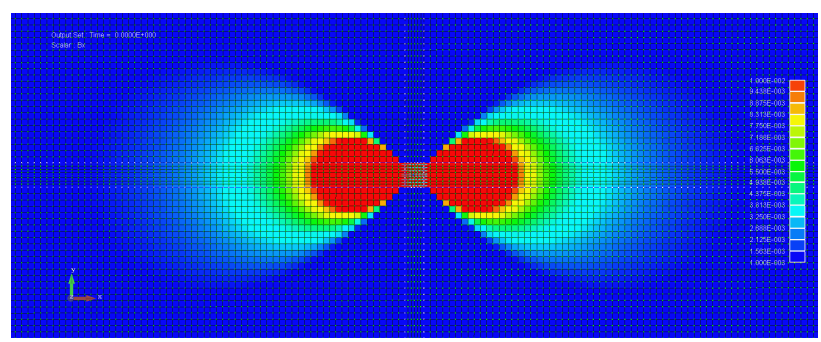

Fig. 13 Analytical results of one magnet

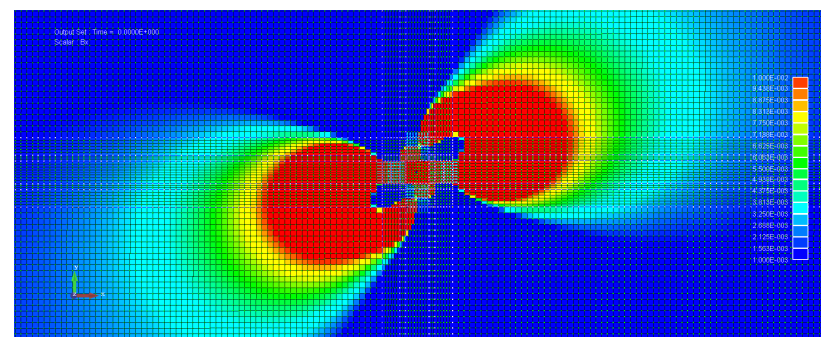

Fig. 14 Analytical results of four magnets

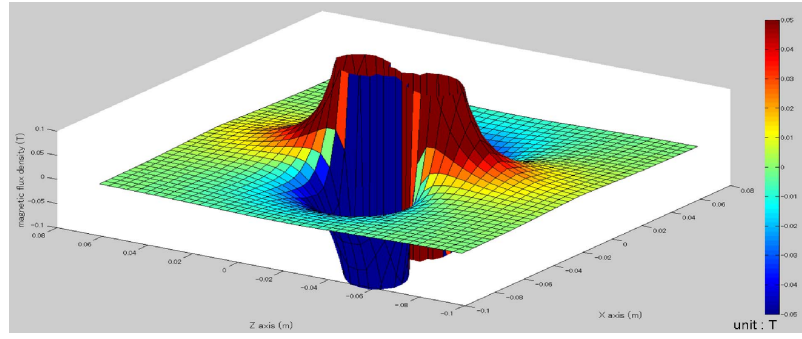

Fig. 15 Analytical results of the o-ring magnet 


\section{Experimental results}

To evaluate the proposed position and posture detection systems, we used high sensitive magnetic field sensor. 3dimensional magnetic field sensor device is shown in Fig. 16. In this experiment, we used this HMC1021Z's by Honey Well as the magnetic field sensor. This sensor is 1-axis. In order to gain the position and posture of the microrobot on a 3dimensions, it is needed to gain the six outputs of the magnetic field, because of the theoretical intensity of the magnetic field is indicated by six parameters. Those parameters are 3 parameters of the position information which are $\mathrm{X}$ and $\mathrm{Y}$ and $\mathrm{Z}$, and 3 parameters of the posture information which are roll and pitch and yaw. Developed position detection device using magnetic field sensor is shown in Fig. 17.

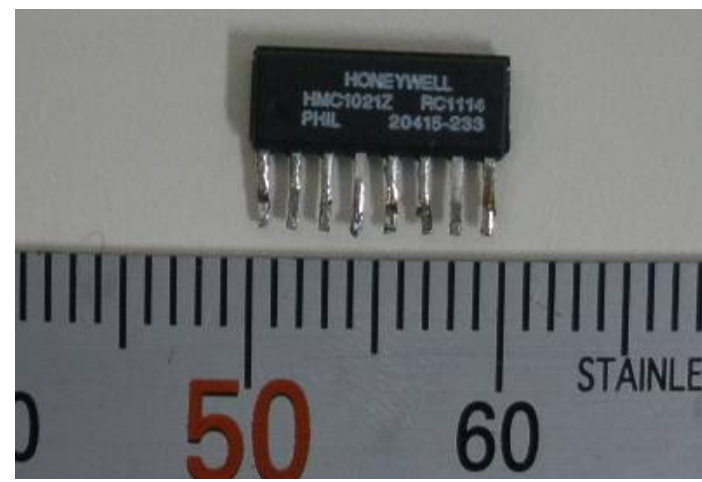

Fig. 16 High sensitive magnetic field sensor

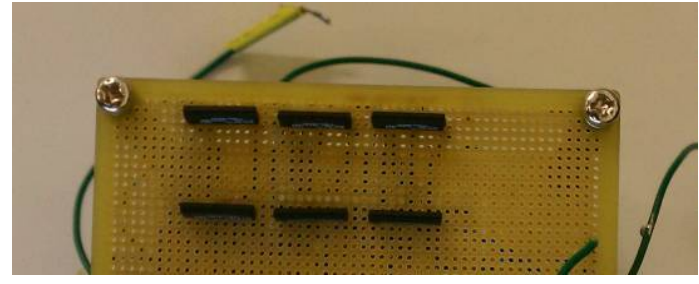

Fig. 17 Set position of the magnetic sensor

Fig. 18 is the result of the output voltage of the magnetic flux density. Theoretical values and measured values are almost identical values. Value of the correlation coefficient is 0.9994 .

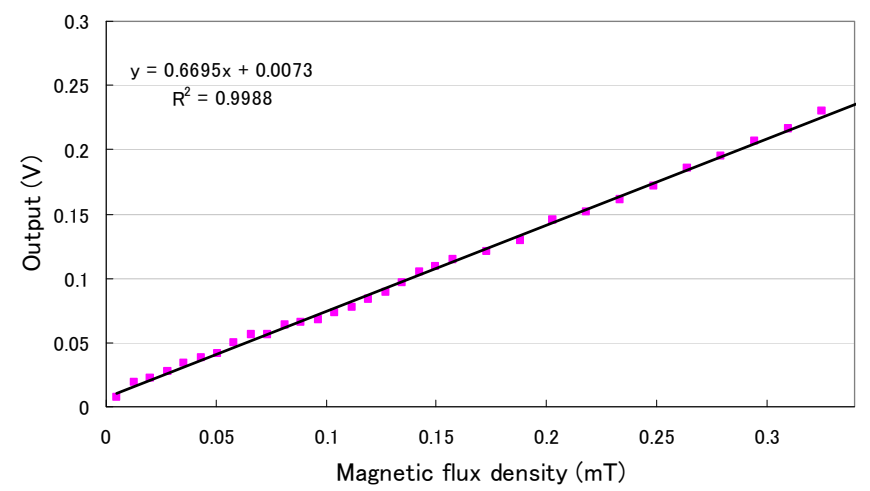

Fig. 18 Characteristic of the magnetic field sensor
We used least square method to do position detection of the microrobot. Equation function of the least square method is indicated in Eq. (7). When this function is the smallest of all, those 6 parameters is the best because of its parameter is similar to true value.

$$
\begin{aligned}
& E(x)=\frac{1}{2}\|e(x)\|^{2}=\frac{1}{2} \sum_{k} e_{k}^{2} \\
& e_{i}=B_{i}-P_{i} \quad(\mathrm{i}=0,1, \sim 5)
\end{aligned}
$$

Where $E$ is equation function of the least square method, $B$ is the experimental values, and $P$ is the theoretical values. $i$ is the number of the sensor. So, total number of $i$ is six.

Fig. 19 is the result of the error when the magnet in the body was separated from the magnetic field sensor. We evaluated it can detect how long it separates from sensor in vertical direction. The result indicated this system can detect at $3-5 \mathrm{~cm}$ in vertical from the sensor. And, from this result, to measure the position of the microrobot has been obtained sufficient accuracy because of the average error is within 6.09 $\mathrm{mm}$.

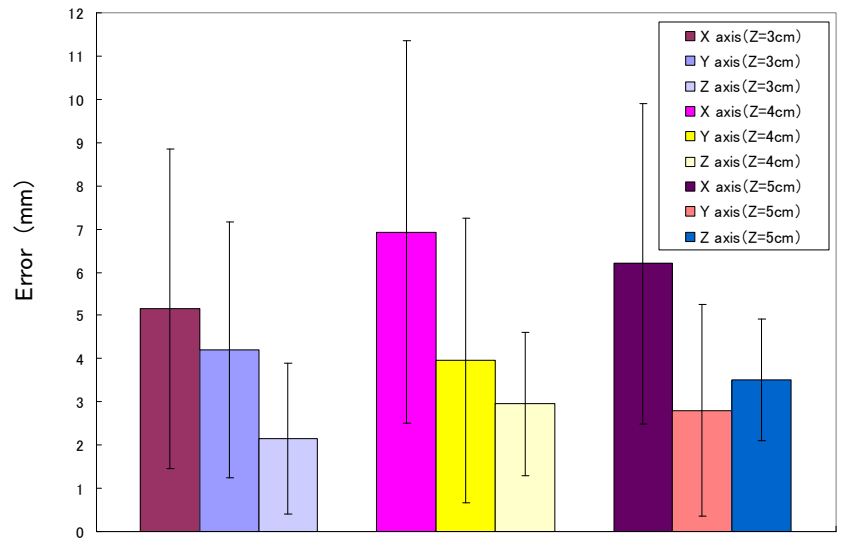

Fig. 19 Experimental results of the position detection error

Finally, we evaluated error of the trajectory between actual positions of the magnet and calculated position using the proposed position detection system. That result is shown in Fig. 20. Green line is the trajectory of the magnet, and red line is the trajectory of the calculated result.

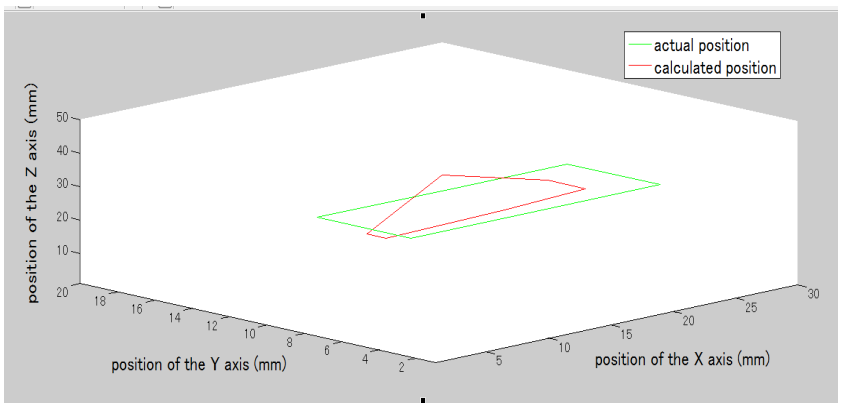

Fig. 20 Calculated result of the trajectory of the magnet 


\section{CONCLUSIONS}

In this paper, to realize locomotion and control of the microrobot, we developed one wireless locomotion systems. The proposed wireless microrobot has two motions by changing the outside magnetic field. When it generates alternating magnetic field, the microrobot can move by the fin motion just like fish. When it generates rotating magnetic field, the microrobot can move by the spiral jet motion. Based on these locomotion, we did experiment of the multi-DOFs locomotion. First, we tried to move in horizontal direction. The microrobot could move forward by fin motion or spiral jet motion. And, the microrobot could move backward by spiral jet motion. Second, we did experiment of the motion in vertical direction. Finally, we evaluated turning left motion and turning right motion to realize the multi-DOFs locomotion.

In addition, we developed a position detection system of the microrobot. We used least square method to solve the inverse problem. When the equation function of the least square method becomes small, we choose that positioning information as true value. To evaluate the error of the proposed position detection system, we did experiment by using 6 magnetic field sensors and program. Based on this experiment, we confirmed that the proposed position detection system can detect position of the microrobot within $50 \mathrm{~mm}$ from sensor in vertical direction, and its average error is within $6.09 \mathrm{~mm}$. In future, we will deal with the control of the direction and moving speed of the microrobot by using this position detection system and CCD camera. Also, it is need to locomotion of the microrobot in actual environment just like in the body of the human. This proposed wireless locomotion has an important role in medical field.

\section{ACKNOWLEDGMENT}

This research is supported by Kagawa University Characteristic Prior Research Fund 2011.

\section{REFERENCES}

[1] Qinxue Pan, Shuxiang Guo, Takuya Okada, "Development of a Wireless Hybrid Microrobot for Biomedical Applications", Proceedings of the 2010 IEEE/RSJ International Conference on Intelligent Robots and Systems, pp.5768-5773, Taipei, Taiwan, Oct. 18-22, 2010.

[2] Baofeng Gao, Shuxiang Guo, Xiufen Ye, "Motion-control Analysis of ICPF- actuated Underwater Biomimetic Microrobots", International Journal of Mechatronics and Automation, Vol. 1, No.2, pp.79-89, 2011.

[3] Qinxue Pan, Shuxiang Guo, Takuya Okada, "A Novel Hybrid Wireless Microrobot", International Journal of Mechatronics and Automation, Vol.1, No.1, pp.60-69, 2011.

[4] K. Mohri, T. Uchiyama, L.P. Shen, C.M. Cai, L.V. Panina, "Sensitive micro magneticsensor family utilizing magneto-impedance (MI) and stress-impedance (SI) effects for intelligent measurements and controls", Sensors and Actuators A 91 (2001), pp. 85-90

[5] S Sudo, S Segawa and T Honda, "Basic Research on the Medical MicroRobot using a Permanent Magnet and Alternating Magnetic Field", Proceedings of Second International Symposium on Aqua BioMechanisms, Honolulu, Hawaii, CD-ROM 1-6,2003.

[6] Yogo Takada, Ryosuke Araki, Yukinobu Nakanishi, Motohiro Nonogaki, Kazuaki Ebita, and Tomoyuki Wakisaka, "Development of Small Fish Robots Powered by Small and Ultra-Light Passive-Type
Polymer Electrolyte Fuel Cells", Journal of Robotics and Mechatronics, Vol.22, No.2, pp.150-157, 2010.

[7] Josep M. Mirats Tur, William Garthwaite, "Robotic Devices for Water Main In-Pipe Inspection: A Survey", Journal of Field Robotics, Volume 27, pp. 491-508, 4, July 2010.

[8] S. Yabukami, S. Hayashi, Y. Tokunaga, T. Kohno, K..I. Arai, and Y. Okazaki, "Development of a Position-Sensing System for a Wireless Magnetic Marker", Journal of the Magnetics Society of Japan (2004) vol.28. pp. 877-885.

[9] M. Toyoda, S. Hayashi, S. Yabukami, M. Ohya, K. Ishiyama, Y. Okazaki, K..I. Arai, "Development of Wireless Magnetic Multi-position Detecting System Using FFT Analysis(Measurement and HighFrequency Device)", J. Magn. Soc. Jpn., 30, pp. 391-395, (2006).

[10] H Park, S Park, E Yoon, B Kim, J Park and S Park, "Paddling based Microrobot for Capsule Endoscopes", IEEE International Conference on Robotics and Automation (ICRA'07), pp. 3377-3382, Roma, Italy, 10-14 April, 2007.

[11] X.D. Wu, W.S. Hou, C.L. Peng et al.., "Wearable magnetic locating and trackingsystem for MEMS medical capsule", Sens. Actuators A 141 (2008), pp. 432-439

[12] Haiming Qi, Jinrui Ye, Xiaohua Zhang, Hongjun Chen, "Wireless tracking and locating system for in-pipe robot", Sens. Actuators A, 159 (2010), pp. 117-125.

[13] Yu Geliang, Bu Xiongzhu, Xiang Chao, Xu Hong, "Design of a GMI magnetic sensor based on longitudinal excitation" Sensors and Actuators A 161 (2010) pp.72-77.

[14] Saman Hosseini, Moein Mehrtash, Mir Behrad,Khamesee, "Design, fabrication and control of a magnetic capsule-robot for the human esophagus", Special issue: 20th ASME Conference on Information Storage and Processing Systems, Santa Clara, CA, USA, 14-15 June 2010, Volume 17, Numbers 5-7 (2011), 1145-1152.

[15] Chungseon Yu, Hyunchul Choi, Jongoh Park, and Sukho Park, "ThreeDimensional Electromagnetic Actuation System for Intravascular Locomotion", Proceedings of IEEE/RSJ International Conference on Intelligent Robots and Systems, pp.540-545, 2009.

[16] Semi Jeong, Hyunchul Choi, Jongho Choi, Chungsun Yu, Jong-oh Park, Sukho Park, "Novel electromagnetic actuation (EMA) method for 3dimensional locomotion of intravascular microrobot", Sensors and Actuators A 157 (2010), pp. 118-125.

[17] Chungseon Yu, Juhyun Kim, Hyunchul Choi, Jongho Choi, Semi Jeong, Kyoungrae Cha, Jong-oh Park, Sukho Park, "Novel electromagnetic actuation system for three-dimensional locomotion and drilling of intravascular microrobot", Sensors and Actuators A 161 (2010), pp. 297-304.

[18] Jongho Choi, Semi Jeong, Kyoungrae Cha, Lulu Qin, Jie Li, Jongoh Park, Sukho Park, "Positioning of Microrobot in a Pulsating Flow using EMA System", Biomedical Robotics and Biomechatronics (BioRob), 2010 3rd IEEE RAS and EMBS International, pp. 588 - 593, September 26-29, 2010

[19] Semi Jeong, Hyunchul Choi, Kyoungrae Cha, Jie Li, Jong-oh Park, Sukho Park, "Enhanced locomotive and drilling microrobot using precessional and gradient magnetic field", Sensors and Actuators A 171 (2011), pp. 429- 435.

[20] V. Schlageter, P.-A. Besse, R.S. Popovic, P. Kucera, "Tracking system with five degrees of freedom using a 2D-array of Hall sensors and a permanent magnet" Sensors and Actuators A 92 (2001), pp.37-42.

[21] M Sendoh, Y Suda, K Ishiyama, K Arai, "Fabrication of Magnetic Actuator for Use in Colon Endoscope", 2003 International Symposium on Micromechatronics and Human Science, 2003.

[22] Shuxiang Guo, Qinxue Pan, "Mechanism and Control of a Novel Type Microrobot for Biomedical Application", Proceedings of the 2007 IEEE International Conference on Robotics and Automation (ICRA2007), Roma, Italy, pp. 187-192, 2007.

[23] Takashi NAGAOKA, Daisuke ICHIKAWA, Akihiko UCHIYAMA, "Development and Application of a Three-dimensional Position Sensor Using a Permanent Magnet", Japanese Society for Medical and Engineering (2004), pp. 100-106. 